\title{
SAKURAI'S OBJECT, V605 AQUILAE, AND FG SAGITTAE: AN EVOLUTIONARY SEQUENCE REVEALED
}

\author{
T. M. LAWLOR \\ Pennsylvania State University, Wilkes-Barre Campus, Lehman, PA 18627; tlawlor@psu.edu \\ AND \\ J. MacDonald \\ Department of Physics and Astronomy, University of Delaware, Newark, DE 19716; jimmacd@udel.edu \\ Received 2002 June 25; accepted 2002 October 4
}

\begin{abstract}
We have completed a grid of stellar evolution calculations to study the behavior of the born-again phenomenon. All our evolutionary sequences begin with a uniform composition $1 M_{\odot}$ star on the pre-main-sequence Hayashi phase and end on the white dwarf cooling track. The effects of combined helium and hydrogen burning and time-dependent convective mixing are included. We artificially vary the mass-loss rate beginning at the peak of the last thermal pulse on the asymptotic giant branch in order to create a range of He-layer masses for the post-asymptotic giant branch (AGB) evolution. We find that a very late thermal pulse occurs in 10\%$15 \%$ of cases. Our models supply an answer to the question of why the born-again stars V4334 Sgr (Sakurai's object) and V605 Aql have a significantly shorter evolutionary timescale than the otherwise similar bornagain star FG Sge. FG Sge has been observed to undergo born-again behavior for more than $120 \mathrm{yr}$, while the other two objects have evolved in a similar way but in less then $10 \mathrm{yr}$. Models with low convective mixing efficiency, $\sim 10^{-4}$, first evolve quickly to the AGB, return to the blue, and then evolve more slowly back to the AGB for a second time before finally returning to the white dwarf cooling track. The difference in evolution timescales can then be explained by proposing that Sakurai's object is evolving to the AGB for the first time but that FG Sge has been observed during its second return to the AGB. Our models allow us to make some testable predictions: (1) Sakurai's object will increase in effective temperature in the next 20-50 yr and will then resemble V605 Aql's present high effective temperature state; (2) V605 Aql will cool back toward the AGB some time in the next 50-70 yr, at which point it will evolve in the same way as has been observed for FG Sge over the last $120 \mathrm{yr}$; and (3) FG Sge will show signs of increasing its effective temperature by about $1500-2000 \mathrm{~K}$ in as soon as 10-20 yr, depending on the metallicity of its progenitor main-sequence star.
\end{abstract}

Subject headings: stars: AGB and post-AGB — stars: evolution stars: individual (Sakurai's object, V605 Aquilae, FG Sagittae) — stars: variables: other

\section{INTRODUCTION}

Stars of initial mass $\lesssim 10.5 M_{\odot}$ evolve onto the asymptotic giant branch (AGB) after completing core helium burning (García-Berro \& Iben 1994; Iben \& MacDonald 1995; Ritossa, García-Berro, \& Iben 1999). Initially helium burns quiescently in a thick shell around the electrondegenerate core. When the helium shell mass is reduced to $\sim 10^{-2} M_{\odot}$, the star continues its evolution through a series of thermally pulses, alternately burning hydrogen and helium in shells (Schwarzschild \& Härm 1965; Weigert 1967). During AGB evolution, the star becomes a longperiod variable. Such stars are observed to lose mass at high rates $\left(10^{-7}\right.$ to $\left.10^{-5} M_{\odot} \mathrm{yr}^{-1}\right)$ that are correlated with the pulsation period. The mass loss creates a slowly expanding circumstellar shell. When the mass of the hydrogen-rich envelope drops below a critical value $\left(\sim 10^{-3} M_{\odot}\right)$, the envelope begins a phase of contraction at constant luminosity. During the contraction the effective temperature increases. This leads to a fast radiatively driven wind that compresses the circumstellar material. The hardening of the stellar radiation with increasing effective temperature leads to ionization of the compressed material, creating a planetary nebula (Kwok 1987).

When thermonuclear reactions cease, the star begins to cool and moves in the H-R diagram to the white dwarf cooling track. However, for stars entering the cooling track with enough helium remaining, a final helium shell flash can occur (Schönberner 1979; Iben 1982). As a result of this shell flash, the star returns to the AGB. The transition is characterized by a 4 mag increase in luminosity and a drop in effective temperature of many tens of thousands of degrees in a very short time, $\sim 5-15$ yr. Additionally, the stellar photosphere becomes deficient in hydrogen, rich in helium, and enriched in carbon, nitrogen, oxygen, and $s$ process materials (Iben \& Livio 1993; Iben \& MacDonald 1995). Iben used the picturesque term "born again" (BA) to describe this behavior. Blöcker \& Schönberner (1997) and Blöcker (2001) prefer the term "very late thermal pulse " (VLTP) to distinguish it from a "late thermal pulse" (LTP), which is a final thermal pulse that occurs when the model is evolving from the AGB to the white dwarf cooling track at approximately constant luminosity, and an "AGB final thermal pulse," which occurs just before the model leaves the AGB.

Because the VLTP occurs in a low-luminosity envelope, the entropy due to radiation is negligible. Unlike the conditions in an AGB star (Iben 1976) or during a LTP, this means that there is only a small entropy barrier to surmount and so the convective zone that is created because of the high fluxes generated by helium burning can penetrate into the hydrogen-rich envelope (Fujimoto 1977).

When the BA phenomenon was first modeled (Iben et al. 1983), it was largely used to explain the chemical 
characteristics of $\mathrm{H}$-deficient planetary nebulae such as Abell 30 and Abell 78. The first observed candidate for a star undergoing a very late helium flash is FG Sge (Iben 1984). It has exhibited the characteristics of born-again behavior over a timescale of about $100 \mathrm{yr}$ (Gonzalez et al. 1998). The discovery of Sakurai's object (V4334 Sgr) in 1996 provided a second example of a post-AGB helium flash but with a timescale of about 6 yr (Duerbeck et al. 2002). V605 Aql, the central star of the planetary nebula Abell 58, is also believed to have undergone a VLTP in 1917 (Clayton \& de Marco 1997). In the earliest calculations of born-again evolution, energy generation from the burning of hydrogen after ingestion into the helium burning convective shell was suppressed to avoid numerical difficulties. The first calculation to include a realistic treatment of combined hydrogen and helium burning plus time-dependent mixing was carried out by Iben \& MacDonald (1995). In their simulation, it took $17 \mathrm{yr}$ for the transition from white dwarf star to AGB star. Once the star had returned to the AGB they stopped their calculation. They suggested that the discrepancy with FG Sge's 100 yr timescale was a result of an incorrect choice of initial parameters. Blöcker (2001) has proposed that FG Sge is the result of a late thermal pulse. The observed hydrogen deficiency (Gonzalez et al. 1998) is achieved by allowing convective overshoot to overcome the barrier to mixing at the hydrogen-helium interface (Herwig et al. 1999). Herwig (2001) finds that the short evolutionary calculation for Sakurai's object can be obtained with a VLTP if the efficiency of element mixing in the He-flash convection zone is reduced by a factor of 100 compared to that predicted from mixing-length theory. Without the reduction in convective efficiency, the evolution timescale is about $350 \mathrm{yr}$. In $\S 2$ we present new VLTP stellar evolution calculations that show a more complex double-loop path in the H-R diagram that is consistent with both the long-timescale evolution of FG Sge and the much quicker evolution of Sakurai's object and V605 Aql. We compare our models to the observations in $\S 3$. Our summary and conclusions are given in $\S 4$.

\section{THEORETICAL MODELS}

\subsection{Description of the Stellar Evolution Program}

The code used is a modified version of that developed by Eggleton $(1971,1972)$. The whole star is evolved by a relaxation method without use of separate envelope calculations. Some of the advantages of this approach are that (1) gravothermal energy generation terms are automatically included for the stellar envelope, (2) mass loss occurs at the stellar surface rather than an interior point of the star, and (3) the occurrence of convective dredge-up of elements produced by nucleosynthesis in the interior to the photosphere is clearly identifiable. Our adaptive mesh technique is similar to that of Winkler, Norman, \& Newman (1984). The advection terms are approximated by second-order upwind finite differences. Convective energy transport is modeled by using the mixing-length theory described by Mihalas (1970). Composition equations for $\mathrm{H},{ }^{3} \mathrm{He},{ }^{4} \mathrm{He},{ }^{12} \mathrm{C},{ }^{14} \mathrm{~N},{ }^{16} \mathrm{O}$, and ${ }^{24} \mathrm{Mg}$ are solved simultaneously with the structure equations. Composition changes due to convective mixing are modeled in the same way as Eggleton (1972) by adding diffusion terms to the composition equations. However, we use a prescription for the diffusion coefficient that differs from that of Eggleton (1972) but is consistent with mixing-length theory (Iben \& MacDonald 1995). The diffusion coefficient is $\sigma_{\text {con }}=\beta \omega_{\text {con }} l$, where $\omega_{\text {con }}$ is the convective velocity, $l$ is the mixing length, and $\beta$ is a dimensionless convective mixing efficiency parameter. For temperatures (in kelvins) above $\log _{10} T=3.84$, we use OPAL radiative opacities (Iglesias \& Rogers 1996). For temperatures below $\log _{10} T=3.78$, we use opacities kindly supplied by $\mathrm{D}$. Alexander and calculated by the method of Alexander \& Ferguson (1994). Between these temperatures, we interpolate between the OPAL and Alexander opacities. Nuclear reaction rates are taken from Fowler, Caughlan, \& Zimmerman (1975), Harris et al. (1983), Nomoto, Thielemann, \& Miyaji (1985), Caughlan \& Fowler (1988), and Arnett (1996), and screening corrections are from Salpeter \& Van Horn (1969) and Itoh et al. (1979). Neutrino loss rates are from Beaudet, Petrosian, \& Salpeter (1967) with modifications for neutral currents (Ramadurai 1976). Plasma neutrino rates are from Haft, Raffelt, \& Weiss (1994). The equation of state is determined by minimization of a model free energy (see, e.g., Fontaine, Graboske \& van Horn 1977) that includes contributions from internal states of all the ionization states of $\mathrm{H}, \mathrm{He}, \mathrm{C}, \mathrm{N}, \mathrm{O}$, and $\mathrm{Mg}$. Electron degeneracy is included by the method of Eggleton, Faulkner, \& Flannery (1973). Coulomb and quantum corrections to the equation of state follow the prescription of Iben, Fujimoto, \& MacDonald (1992), with the Coulomb free energy updated to use the results of Stringfellow, DeWitt, \& Slattery (1990). Pressure ionization is included in a thermodynamically consistent manner by use of a hardsphere free-energy term (Boublik 1970; Mansoori et al. 1971; Lebowitz, Helfand, \& Praestgaard 1965).

Mass loss is included by using a scaled Reimers (1975) mass-loss law,

$$
\dot{M}=-\eta 4 \times 10^{-13} \frac{L}{L_{\odot}} \frac{R}{R_{\odot}}\left(\frac{M}{M_{\odot}}\right)^{-1} M_{\odot} \mathrm{yr}^{-1},
$$

for cool stars $\left(T_{\text {eff }} \leq 10^{4} \mathrm{~K}\right)$ and an approximation to the theoretical result of $\bar{A}$ bbott (1982),

$$
\dot{M}=-1.2 \times 10^{-15} \frac{Z}{Z_{\odot}}\left(\frac{L}{L_{\odot}}\right)^{2}\left(\frac{M_{\text {eff }}}{M_{\odot}}\right)^{-1} M_{\odot} \mathrm{yr}^{-1}
$$

for hot stars $\left(T_{\text {eff }} \geq 10^{4} \mathrm{~K}\right)$.

\subsection{Construction of Initial Models for VLTP Studies}

We have evolved $1 M_{\odot}$ stars of metallicity $Z=0.001$, $0.004,0.01$, and 0.02 from the pre-main-sequence Hayashi phase to the white dwarf cooling track. Based on the findings of Wood \& Cahn (1977), Kudritzki \& Reimers (1978), and Jimenez et al. (1995), we adopt a value $\eta=0.35$ for the mass-loss efficiency parameter. With our choice of initial mass and mass-loss rate, the final remnant masses are close to the typical masses determined for DA white dwarfs (Bragaglia, Renzini, \& Bergeron 1995; Vennes 1999), H-rich central stars of planetary nebulae (CSPN; Napiwotzki 1999), DO white dwarfs (Dreizler \& Werner 1996), and the PG 1159 stars (Werner et al. 1996; Dreizler \& Heber 1998). In Figure 1 we show a representative $\mathrm{H}-\mathrm{R}$ diagram for $Z=0.001$.

To generate a grid of models suitable for investigation of the born-again phenomenon, we vary the mass-loss rate starting from the peak of the last AGB thermal pulse by choosing different values for the mass-loss parameter, $\eta$. This gives a complete range of the possible helium layer 


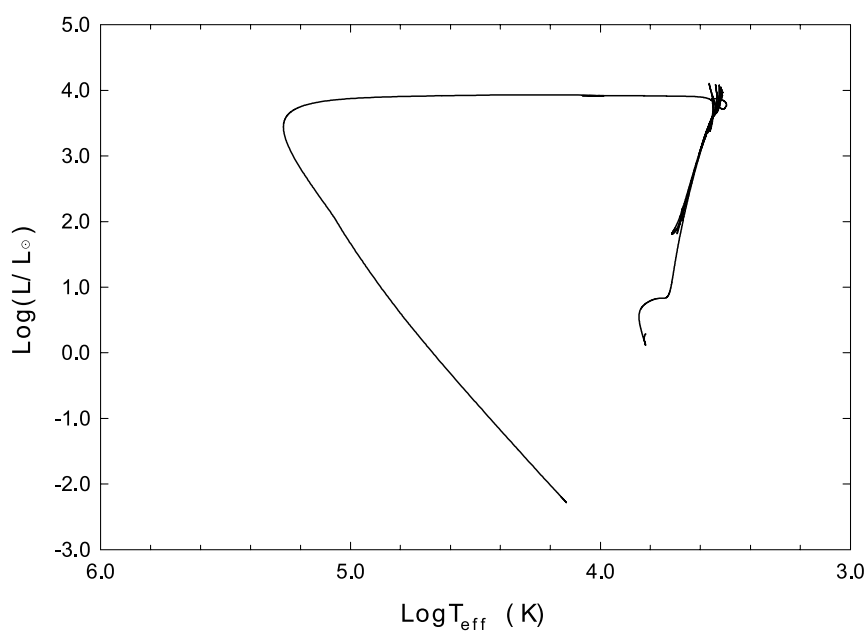

FIG. 1.-Representative H-R diagram for $Z=0.001$

masses at the point when the star leaves the asymptotic giant branch for the first time. A VLTP occurs in $10 \%-15 \%$ of post-AGB stars.

In Table 1 we summarize some general properties of models. The first two columns in the table give the metallicity and the Reimers' mass-loss parameter. The next columns are the stellar mass, the stellar age, the mass above the hydrogen discontinuity, and the mass above the helium discontinuity, all at the time of departure from the normal AGB, which we define as occurring at the last maximum in radius. The next two columns are the masses of hydrogen and helium in the final white dwarf remnant. The type of post-AGB behavior is given in the final column. Here stable indicates that there are no more thermal pulses after leaving the AGB, H-rich WD indicates that the evolutionary endpoint will be a DA white dwarf, VLTP and LTP indicate that there is a very late thermal pulse or a late thermal pulse after leaving the AGB, He-rich WD indicates that the evolu- tionary endpoint is likely to be a DO/DB white dwarf (provided that mass loss after the VLTP is sufficient to remove the small remaining amount of hydrogen), AFTP indicates that a helium flash occurs just at the point of departure from the AGB, and additional AGB TP indicates that a lowered Reimers' mass-loss efficiency parameter results in an additional AGB thermal pulse that is not found with the canonical value of 0.35 .

\subsection{Results of the Evolutionary Calculations: Dependence on Mixing Efficiency}

Herwig (2001) has shown that the evolutionary timescale of VLTP stars strongly depends on the value chosen for the convective mixing efficiency parameter $\beta$. To explore the dependence of our results on this parameter, we have calculated for the $Z=0.02, \eta=0.06$ case evolutionary sequences with $\beta=10^{-4}, 10^{-3}$, and $10^{-2}$. We begin by describing the results of these three evolutionary calculations because they determine our choice of $\beta$ for the other VLTP sequences.

For $\beta=10^{-4}$, the surface luminosity increases by more than 4 mag in a few months, during which the temperature also increases to a first maximum of about $50,000 \mathrm{~K}$ and the radius increases to about 20 white dwarf radii. The luminosity increases, then slows, and because the radius continues to increase, the effective temperature decreases to about $5500 \mathrm{~K}$ over a period of about $2 \mathrm{yr}$. After about $10 \mathrm{yr}$, the star's radius has increased to about $10^{13} \mathrm{~cm}$. About $25 \mathrm{yr}$ after the initial rapid increase in luminosity, the radius decreases very quickly back to about 20 white dwarf radii and then slowly increases again, reaching giant dimensions on a timescale of order $300 \mathrm{yr}$. The luminosity drops by about a factor of 10 at the time of rapid shrinking of the star, and hence the temperature increases rapidly to a second maximum of about $80,000 \mathrm{~K}$ at this time. As the radius increases for the second time, the luminosity returns to maximum and the effective temperature again decreases to about $6000 \mathrm{~K}$. Finally, after about $800 \mathrm{yr}$ from the initial flash, the radius begins to decrease. Before embarking on

TABLE 1

Dependence of Post-Agb Behavior on Metallicty and Reimers' Mass-Loss Parameter

\begin{tabular}{|c|c|c|c|c|c|c|c|c|}
\hline$Z$ & $\begin{array}{c}\text { Reimers' Mass-Loss } \\
\text { Parameter }\end{array}$ & $\begin{array}{l}M_{\text {star }} \\
\left(M_{\odot}\right)\end{array}$ & $\begin{array}{l}\text { Age } \\
(\mathrm{Gyr})\end{array}$ & $\begin{array}{c}M_{\mathrm{HS}} \\
\left(10^{-2} M_{\odot}\right)\end{array}$ & $\begin{array}{c}M_{\mathrm{HeS}} \\
\left(10^{-2} M_{\odot}\right)\end{array}$ & $\begin{array}{c}M_{\mathrm{HWD}} \\
\left(10^{-4} M_{\odot}\right)\end{array}$ & $\begin{array}{c}M_{\mathrm{HeWD}} \\
\left(10^{-2} M_{\odot}\right)\end{array}$ & Post-AGB Behavior \\
\hline \multirow[t]{4}{*}{$0.001 \ldots}$. & 0.35 & 0.621 & \multirow[t]{4}{*}{7.41} & 1.16 & 2.21 & 0.61 & 1.09 & Stable; H-rich WD \\
\hline & 0.163 & 0.624 & & 1.37 & 2.45 & $4.4 \times 10^{-4}$ & 0.69 & LTP \\
\hline & 0.140 & 0.624 & & 1.41 & 2.50 & 0.61 & 0.77 & AFTP \\
\hline & $0.01-0.13$ & $0.625-0.628$ & & $1.41-1.67$ & $2.49-2.84$ & $0.59-0.88$ & $0.80-0.98$ & $\begin{array}{l}\text { Additional AGB TPs; } \\
\text { Stable; H-rich WD }\end{array}$ \\
\hline & $0.12-0.15$ & 0.599 & \multirow{3}{*}{8.88} & $1.08-1.11$ & $2.18-2.23$ & $2.0 \times 10^{-3}-2.2 \times 10^{-3}$ & $0.83-0.86$ & VLTP; He-rich WD \\
\hline & 0.10 & 0.600 & & 1.16 & 2.28 & 0.40 & 0.86 & LTP \\
\hline & $0.07-0.08$ & 0.600 & & $1.19-1.21$ & $2.32-2.35$ & $0.81-1.81$ & $0.91-0.97$ & AFTP \\
\hline \multirow[t]{4}{*}{$0.01 \ldots \ldots$} & $0.18-0.35$ & $0.571-0.573$ & \multirow[t]{4}{*}{11.4} & $0.730-0.886$ & $0.94-1.15$ & $0.91-0.94$ & $1.22-1.40$ & Stable; H-rich WD \\
\hline & $0.12-0.17$ & $0.573-0.574$ & & $0.903-0.952$ & $1.18-1.25$ & $7.0 \times 10^{-3}-8.0 \times 10^{-3}$ & $0.89-0.90$ & VLTP; He-rich WD \\
\hline & 0.11 & 0.574 & & 0.967 & 1.27 & 0.30 & 0.90 & LTP \\
\hline & $0.08-0.10$ & $0.575-0.577$ & & $0.973-1.11$ & $1.29-1.46$ & $0.88-0.91$ & $0.97-1.07$ & AFTP \\
\hline \multirow{2}{*}{$0.02 \ldots \ldots$} & 0.04 & 0.567 & \multirow{2}{*}{13.9} & 0.594 & 1.64 & 0.81 & 1.05 & LTP \\
\hline & 0.01 & 0.568 & & 0.627 & 1.25 & 0.79 & 1.18 & AFTP \\
\hline
\end{tabular}




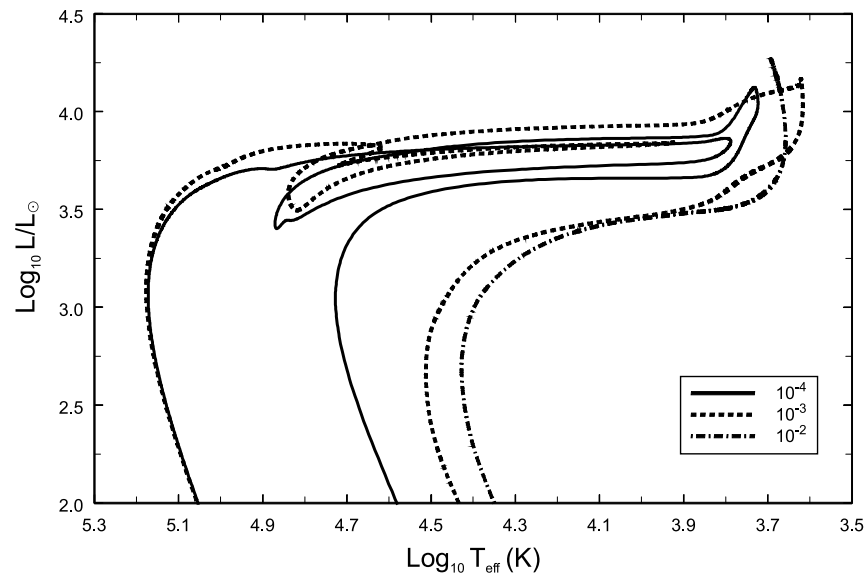

FIG. 2.-Dependence of the H-R diagram of VLTP models on the convective efficiency parameter, $\beta$.

the white cooling track, the effective temperature reaches a third maximum of $160,000 \mathrm{~K}$ at about $20,000 \mathrm{yr}$ after the initial flash. The evolutionary path in the H-R diagram, shown in Figure 2, has a double-loop structure.

The double loop in the H-R diagram is a result of the penetration of the He-flash-driven convection zone into H-rich layers. When protons are convected to hot enough regions they are captured by ${ }^{12} \mathrm{C}$ nuclei. Ingestion of protons by the convection zone continues until the rate of energy generation by proton captures becomes comparable to that from helium burning. The entropy produced by proton captures forces the splitting of the convective zone into two distinct convective zones: one powered by hydrogen burning and the other by helium burning (Iben \& MacDonald 1995; see also Hollowell, Iben, \& Fujimoto 1990). The initial expansion of the envelope is powered by the H-burning shell. When this dies out, the envelope initially contracts. The second expansion of the envelope is powered by the continuing He-burning shell. As this dies out, the envelope contracts again and the star returns to the white dwarf domain.

As a result of convective dredge up of material processed by $\mathrm{CNO}$ cycling and helium burning, the composition of the envelope can undergo significant changes as the star evolves. Mass loss also plays a role in uncovering nuclear processed material. The degree of mixing of processed material up to the surface and the concomitant mixing of hydrogen down to the burning region strongly depend on the mixing efficiency. For $\beta=10^{-4}$, mixing is relatively weak and composition gradients remain in the envelope throughout much of the evolution, even when the envelope is fully convective. When the star reaches red giant dimensions for the first time after the VLTP, the mass fractions at the photosphere are $X=0.35, \quad Y=0.48, Z_{\mathrm{C}}=0.12$, and $Z_{\mathrm{O}}=0.04$. However in the following phase, in which the star shrinks and increases in effective temperature, the hydrogen mass fraction increases owing to the existence of a composition gradient and the increase in optical depth to a given mass level. When the star returns to giant dimensions for the second time, the hydrogen mass fraction decreases again and the composition near the end of this phase is $X=0.45, Y=0.43, Z_{\mathrm{C}}=0.08$, and $Z_{\mathrm{O}}=0.03$. As the star evolves to the blue, mass loss occurs at a large enough rate to uncover deeper, more highly processed layers. When the star reaches the white dwarf cooling track, the photospheric abundances are $X=0.06, \quad Y=0.57, \quad Z_{\mathrm{C}}=0.26$, and $Z_{\mathrm{O}}=0.07$, and the total mass of hydrogen is $2 \times 10^{-6} M_{\odot}$.

For $\beta=10^{-3}$, evolution initially occurs at a rate similar to the case with $\beta=10^{-4}$, but the evolution after the first return to the blue is much quicker. The time from the second maximum in temperature to the second minimum is approximately $25 \mathrm{yr}$, compared to $500 \mathrm{yr}$ for the case with $\beta=10^{-4}$. The minimum effective temperature is higher, 8300 compared to $6200 \mathrm{~K}$, and the time spent cooler than $10,000 \mathrm{~K}$ is much shorter, 20 compared to $500 \mathrm{yr}$. The degree of convective dredge up of heavy elements and depletion of hydrogen is greater. When the star reaches the white dwarf cooling track, the photospheric abundances are $X=0.02, Y=0.55, Z_{\mathrm{C}}=0.28$, and $Z_{\mathrm{O}}=0.08$, and the total mass of hydrogen is $3 \times 10^{-7} M_{\odot}$. We attribute the shorter lifetime of the second giant phase to the greater mean molecular weight in the envelope that results from the higher convective efficiency. The structure of the star is essentially a degenerate $\mathrm{CO}$ core with a helium-burning shell and a hydrogen-depleted envelope. For the core masses of our models, such stars do not become red giants (Trimble \& Paczyński 1973; Weiss 1987). The star spends about the same time burning helium as the $\beta=10^{-4}$ model, but does it at higher effective temperature.

For $\beta=10^{-2}$, the initial evolution is similar to that with $\beta=10^{-3}$. However, the even greater convective efficiency causes complete homogenization of the envelope on the first return to the giant branch. The envelope abundances at this stage are $X=0.04, Y=0.55, Z_{\mathrm{C}}=0.28$, and $Z_{\mathrm{O}}=0.08$. Slightly higher opacities near $T=2 \times 10^{5} \mathrm{~K}$ associated with the increased carbon abundance are enough to make the luminosity very close to the Eddington limit. The densities in this region are low enough that convection is not completely efficient. The radiation pressure gradient is more than enough to balance gravity. Hence, our assumption of hydrostatic equilibrium requires that the gas-pressure gradient be opposite to that of the radiation pressure (Asplund 1998). This causes a density inversion, which is clearly seen in Figure 3, where we compare the envelope structure of the $\beta=10^{-4}$ and the $\beta=10^{-2}$ models at a similar stage of evolution near the time of highest luminosity on the (first) red

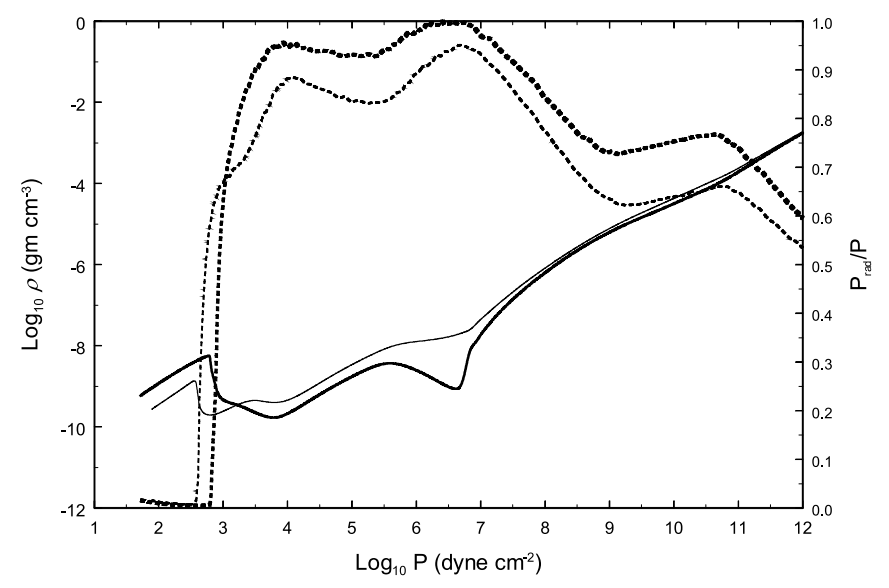

FIG. 3.-Density, $\rho$ (solid lines), and the ratio of radiation pressure to total pressure, $P_{\mathrm{rad}} / P$ (broken lines), plotted against total pressure for the $\beta=10^{-4}$ (thin lines) and $\beta=10^{-2}$ models (thick lines) near the time of maximum radius. The density inversion that results from the radiative flux locally exceeding the Eddington limit can clearly be seen for the $\beta=10^{-2}$ case near $\log P=6$. 
giant branch. (The density inversion near $P=500 \mathrm{dyn}^{-2}$ is due to the change in molecular weight in an ionization zone.) As the density decreases, convection becomes less efficient, and this causes a steeper radiation pressure gradient that in turn requires a steeper gas pressure gradient to maintain hydrostatic equilibrium. This leads to an unstable situation that prevents continuation of the calculation. There is some evidence for instability in the observations. Asplund et al. (1997) find that the photospheric parameters derived for Sakurai's object from analyses of spectra obtained in 1996 May and October are not consistent with the constant luminosity deduced from photometry. As they point out, one possible resolution of this inconsistency is that hydrostatic equilibrium was not applicable in May because of dynamical expansion. They also find that with the May parameters, the star's luminosity is at the classical Eddington limit.

Because the timescales with lower $\beta$ are similar to the evolutionary timescales of FG Sge and Sakurai's object (see $\S 3.3$ for further explanation), and because of the breakdown of the hydrostatic approximation after the first return to the AGB for $\beta \gtrsim 10^{-2}$, we choose $\beta=10^{-4}$ for the rest of the calculations.

\subsection{Results of the Evolutionary Calculations: Dependence on Metallicity}

A representative $\mathrm{H}-\mathrm{R}$ diagram for a VLTP is shown in Figure 4. In the top plot, we show the evolution from the end of the AGB to the white dwarf cooling track. The details of the double-loop evolution are shown in the bottom plot. The times between the labeled points are given in Table 2 for models of different metallicities. Point A corresponds to the beginning of the helium-flash-driven expansion, and point $\mathrm{B}$ corresponds to the time of peak nuclear energy generation. Points $\mathrm{C}$ and $\mathrm{D}$ are at extrema in temperature, and
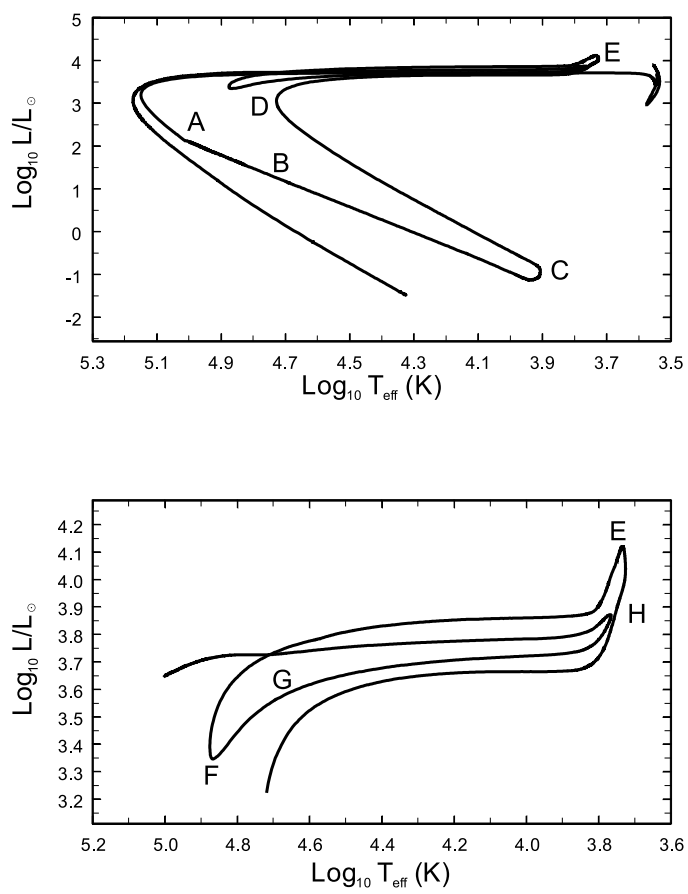

FIG. 4.-Representative H-R diagram for a VLTP
TABLE 2

Timescales for the Very Late Flash

\begin{tabular}{|c|c|c|c|c|c|c|c|}
\hline \multirow{2}{*}{$\begin{array}{c}\text { Metallicity } \\
(Z)\end{array}$} & \multicolumn{7}{|c|}{$\begin{array}{c}\Delta t \\
(\mathrm{yr})\end{array}$} \\
\hline & A-B & $\mathrm{B}-\mathrm{C}$ & C-D & D-E & E-F & $\mathrm{F}-\mathrm{G}$ & $\mathrm{G}-\mathrm{H}$ \\
\hline 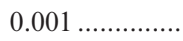 & 6100 & 0.19 & 0.16 & 4.2 & 16 & 125 & 175 \\
\hline $0.004 \ldots \ldots \ldots \ldots$ & 14000 & 0.032 & 0.10 & 6.1 & 19 & 150 & 250 \\
\hline $0.010 \ldots \ldots \ldots \ldots . . .$. & 1100 & 0.065 & 0.18 & 9.5 & 31 & 240 & 280 \\
\hline $0.020 \ldots \ldots \ldots \ldots . . . . .$. & 5900 & 0.12 & 0.25 & 12 & 33 & 290 & 250 \\
\hline
\end{tabular}

points $\mathrm{E}, \mathrm{F}$, and $\mathrm{H}$ are at extrema in luminosity. At point $\mathrm{G}$, the helium-burning luminosity has a local minimum.

Specific remarkable changes in extremely short timescales occur between points $\mathrm{B}$ and $\mathrm{E}$, where the stellar model completes its first return to the AGB. During the time between B and $\mathrm{D}$ both helium burning and hydrogen burning are extremely intense $\left(>10^{6} L_{\odot}\right)$. Following point $\mathrm{D}$, a star will spend approximately $5-10$ yr to get to cooler temperatures, during which time the star grows to giant size, hydrogen burning is mostly extinguished, and helium burning is diminished to about $3000-6500 L_{\odot}$, depending on metallicity. The star then traverses its path backward (E to F) on a timescale of about 10-50 yr, contracting to less then solar size. It remains on the hot side of the H-R diagram for 50 $100 \mathrm{yr}$. At point G, about 130-270 yr following turning point $F$, helium burning increases again to about 7500 $14,000 L_{\odot}$, and within an additional 110-230 yr the star will complete its second return to the AGB, reaching giant size for the last time. Following this stage, helium burns at an almost constant rate, while the star contracts to become a CSPN for the second time. A second ionized nebula is formed with an observably smaller radius than the first one created as much as $3000-5000 \mathrm{yr}$ earlier. After point D, there is a correlation between the timescales and the metallicity. Higher metallicity leads to slower evolution.

During the double loop, extremely rapid and sweeping changes in surface chemical composition also take place. Over this short period, the surface of the star becomes deficient in hydrogen and rich in helium. It also becomes enriched in carbon, oxygen, and nitrogen. In Table 3 we compare in mass fractions the amount of $\mathrm{H}, \mathrm{He}, \mathrm{C}, \mathrm{O}$, and $\mathrm{N}$ before and after a very late helium flash and also include the time duration for which these changes take place. Also compared is the change in effective temperature, luminosity, and radius.

\section{COMPARISONS BETWEEN OBSERVATIONS AND MODEL CALCULATIONS}

\subsection{Summary of Observations}

Three directly observed objects that exhibit behavior consistent with the born-again scenario are Sakurai's object (=V4334 Sgr), FG Sge, and V605 Aql. Sakurai's object (SO) was discovered in 1996 as a star of 11 mag (Nakano, Benetti, \& Duerbeck 1996). Prediscovery observations show that it began to brighten in late 1994 or early 1995 . It has increased in visual luminosity by as much as 4 mag and decreased significantly in temperature within the last $6 \mathrm{yr}$ (Duerbeck et al. 1997). FG Sge has been continuously observed to be brightening and cooling since the late $1800 \mathrm{~s}$ 
TABLE 3

Surface Parameters before and after the Very Late Helium Flash

\begin{tabular}{|c|c|c|c|c|c|c|c|c|c|c|c|c|}
\hline \multirow[b]{2}{*}{ PARAMETER } & \multicolumn{4}{|c|}{ Before Flash (at A) } & \multicolumn{4}{|c|}{ FIrSt RETURN to AGB (at E) } & \multicolumn{4}{|c|}{ SECONd RETURN to AGB (at H) } \\
\hline & (1) & (2) & (3) & (4) & (1) & $(2)$ & (3) & (4) & (1) & (2) & (3) & (4) \\
\hline Initial $Z \ldots \ldots \ldots \ldots$ & 0.001 & 0.004 & 0.01 & 0.02 & 0.001 & 0.004 & 0.01 & 0.02 & 0.001 & 0.004 & 0.01 & 0.02 \\
\hline $\mathrm{H}, \ldots \ldots \ldots \ldots \ldots \ldots$ & 0.699 & 0.709 & 0.702 & 0.675 & 0.680 & 0.478 & 0.389 & 0.355 & 0.142 & 0.404 & 0.442 & 0.514 \\
\hline He................. & 0.287 & 0.281 & 0.286 & 0.304 & 0.304 & 0.453 & 0.472 & 0.475 & 0.726 & 0.502 & 0.444 & 0.397 \\
\hline $\mathrm{C} \ldots \ldots \ldots \ldots \ldots \ldots$ & 0.0094 & 0.0043 & 0.0020 & 0.0030 & 0.0093 & 0.0451 & 0.0940 & 0.116 & 0.0841 & 0.0638 & 0.0765 & 0.0547 \\
\hline $\mathrm{N} \ldots \ldots \ldots \ldots \ldots \ldots$ & 0.0004 & 0.0005 & 0.0010 & 0.0018 & 0.0020 & 0.0054 & 0.0043 & 0.0064 & 0.0149 & 0.0059 & 0.0040 & 0.0052 \\
\hline $\mathrm{O} \ldots \ldots \ldots \ldots \ldots \ldots$ & 0.0028 & 0.0028 & 0.0049 & 0.0101 & 0.0030 & 0.0138 & 0.0312 & 0.0333 & 0.0229 & 0.0262 & 0.0187 & 0.0204 \\
\hline Other metals ... & 0.0010 & 0.0014 & 0.0030 & 0.0053 & 0.0012 & 0.0047 & 0.0090 & 0.0141 & 0.0098 & 0.0062 & 0.0079 & 0.004 \\
\hline $\log T_{\text {eff }}(\mathrm{K}) \ldots \ldots$ & 5.0553 & 5.0179 & 5.0145 & 5.0127 & 3.8434 & 3.7799 & 3.7515 & 3.7341 & 4.4944 & 3.8019 & 3.7539 & 3.7671 \\
\hline $\log L / L \odot \ldots \ldots \ldots$ & 1.9972 & 1.9225 & 2.0793 & 2.1308 & 4.1054 & 4.1778 & 4.1475 & 4.1216 & 4.1053 & 4.0287 & 3.9447 & 3.8727 \\
\hline $\log R(\mathrm{~cm}) \ldots \ldots$. & 9.2531 & 9.2905 & 9.3757 & 9.4051 & 12.731 & 12.894 & 12.936 & 12.958 & 11.429 & 12.776 & 12.829 & 12.767 \\
\hline
\end{tabular}

(Kipper 1996; Gonzalez et al. 1998), and V605 Aql is believed to have flashed in about 1917, reaching its peak only 2 yr later (Clayton \& de Marco 1997). All three objects have increased in luminosity, decreased in temperature, and experienced significant changes in chemical composition (see, e.g., Asplund et al. 1997; Kerber et al. 1999; Asplund et al. 1999 [for Sakurai's object]; van Genderen \& Gautschy 1995; Gonzalez et al. 1998; Langer, Kraft, \& Anderson 1974 [for FG Sge]; Clayton \& de Marco 1997; Harrison 1996; Bond et al. 1993; Kimeswenger et al. 2000; Duerbeck et al. 2002 [for V605 Aql]).

\subsubsection{Sakurai's Object (V4334 Sgr)}

Six-color broadband photometry of SO was carried in 1996, as described by Duerbeck et al. (1997). The authors calculate the luminosity of SO from their measurements of $m_{v}$, an estimate of distance to the object, and bolometric corrections for giants of similar surface temperatures. They determine the effective temperature by comparing observed color indices, corrected for reddening, $\mathrm{CE}=0.53$, to synthetic colors for various temperatures. Data taken from Duerbeck et al. are summarized in Table 4.

Spectra of SO obtained in 1996 by Asplund et al. (1997), Shetrone \& Keane (1997), Kipper \& Klochova (1997), and others have been analyzed in detail by Asplund et al. (1999). The temperatures determined by spectral analysis are in good agreement with those from the photometry. Composition analysis shows that the stellar surface is deficient in hydrogen and enriched in many heavy elements, including $s$-process nuclei. Furthermore the hydrogen abundance decreased over the period from 1996 May-October from $X \sim 0.007$ to about 0.0007 , but the helium abundance

TABLE 4

Summary of SO Data From DUerbeck ET AL. (1997)

\begin{tabular}{|c|c|c|c|}
\hline $\mathrm{JD} 2,450,000+$ & $\begin{array}{l}T_{\text {eff }} \\
(\mathrm{K})\end{array}$ & $\log L / L_{\odot}$ & $\begin{array}{c}\log R \\
(\mathrm{~cm})\end{array}$ \\
\hline $136 \ldots \ldots \ldots \ldots \ldots \ldots$ & 8100 & 3.82 & 12.46 \\
\hline 144 & 8000 & 3.84 & 12.48 \\
\hline 173 & 7900 & 3.86 & 12.50 \\
\hline $196 \ldots$ & 7750 & 3.895 & 12.53 \\
\hline 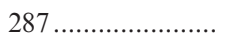 & 7250 & 3.905 & 12.60 \\
\hline 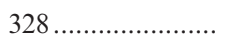 & 7100 & 3.95 & 12.64 \\
\hline $350 \ldots \ldots \ldots \ldots \ldots \ldots$ & 7000 & 3.97 & 12.66 \\
\hline $550 \ldots \ldots \ldots \ldots \ldots$ & 6000 & 4.00 & 12.84 \\
\hline
\end{tabular}

remained fairly constant, $Y \sim 0.2$. The composition determined for SO closely resembles that of the RCrB star V854 Cen (Asplund et al. 1998a).

\subsection{2. $F G S g e$}

FG Sge, the central star of the planetary nebula He 1-5, has slowly increased in visual brightness over the last $100 \mathrm{yr}$ or so, from $m_{\mathrm{pg}}=13.6$ in 1894 to $B=9.6$ in 1965 (Herbig \& Boyarchuk 1968). Its spectral type has also changed significantly, from B5 Ia in 1955, to A5 Ia in 1967 (Herbig \& Boyarchuk 1968), and to G2 Ia in the mid-1970s (Smolinski, Climenhaga, \& Kipper 1976). In the 1980s, its spectral type remained constant near F6 (Montesinos et al. 1990). Although its visual brightness increased significantly, the bolometric magnitude remained almost constant (Wentzel \& Fürtig 1967). In 1992 FG Sge's visual brightness declined rapidly by about 5 mag (Arkhipova 1994). IR observations indicated the formation of a dusty shell (Woodward et al. 1993), which suggests that the visual fading is due to dust condensation. Since 1992 there have been many more rapid fadings and recoveries, similar to those of $\mathrm{R} \mathrm{CrB}$ stars (Jurcsik 1993).

FG Sge's surface abundances have also changed dramatically. In the 1960s it had approximately solar abundances, but in the early 1970s lines of rare earth elements suddenly appeared and rapidly strengthened (Langer et al. 1974). Gonzalez et al. (1998) obtained spectra of FG Sge from 1992 June to 1996 December. They find that the abundances of carbon, scandium, and the rare earth elements are enhanced relative to the Sun and relative to the abundances found by Langer et al. (1974). They also find evidence that hydrogen is deficient by $2-3$ dex. These findings support the idea that FG Sge may be a newborn R CrB star (Gonzalez et al. 1998).

Van Genderen \& Gautschy (1995) have attempted to reconstruct from photometric observations the evolutionary track taken by FG Sge in the H-R diagram for the years 1880-1992. However, Gonzalez et al. (1998) have pointed out that estimates of $T_{\text {eff }}$ from colors are not likely to be reliable after the early 1970s because of the increased line blanketing from the rare earth elements. Montesinos et al. (1990) monitored FG Sge in the ultraviolet and the infrared from 1982 to 1989. They find that an increase in effective temperature occurred sometime after about 1980 and that the star had the same spectral type in 1989 as it did in 1982. Their International Ultraviolet Explorer observations indi- 
TABLE 5

Summary of FG Sge Data From van Genderen \& Gautschy (1995) AND MONTESINOS ET AL. (1990)

\begin{tabular}{cccc}
\hline \hline Date & $\begin{array}{c}\log T_{\text {eff }} \\
(\mathrm{K})\end{array}$ & $\log L / L_{\odot}$ & $\begin{array}{c}\log R \\
(\mathrm{~cm})\end{array}$ \\
\hline \multicolumn{4}{c}{ van Genderen \& Gautschy $(1995)$} \\
\hline $1880 \ldots \ldots \ldots \ldots$ & 4.65 & 3.47 & \\
$1890 \ldots \ldots \ldots \ldots$. & 4.615 & 3.43 & 10.79 \\
$1900 \ldots \ldots \ldots \ldots$ & 4.578 & 3.48 & 10.84 \\
$1910 \ldots \ldots \ldots \ldots$. & 4.540 & 3.58 & 10.95 \\
$1920 \ldots \ldots \ldots \ldots$ & 4.490 & 3.72 & 11.07 \\
$1930 \ldots \ldots \ldots \ldots$. & 4.390 & 3.70 & 11.26 \\
$1940 \ldots \ldots \ldots \ldots$. & 4.250 & 3.64 & 11.43 \\
$1950 \ldots \ldots \ldots \ldots$. & 4.190 & 3.86 & 11.69 \\
$1955 \ldots \ldots \ldots \ldots$. & 4.165 & 3.88 & 11.92 \\
$1960 \ldots \ldots \ldots \ldots$. & 4.035 & 3.78 & 11.99 \\
$1962.5 \ldots \ldots \ldots$. & 3.997 & 3.98 & 12.18 \\
$1965 \ldots \ldots \ldots \ldots$. & 3.965 & 4.05 & 12.36 \\
$1967.5 \ldots \ldots \ldots$. & $3.943-3.950$ & 4.06 & 12.46 \\
$1970 \ldots \ldots \ldots \ldots$. & $3.858-3.895$ & 4.02 & 12.51 \\
$1972.5 \ldots \ldots \ldots$. & $3.773-3.835$ & 4.06 & 12.66 \\
$1975 \ldots \ldots \ldots \ldots$. & $3.727-3.795$ & 4.05 & 12.85 \\
$1980 \ldots \ldots \ldots \ldots$. & $3.688-3.755$ & 4.08 & 12.94 \\
$1985 \ldots \ldots \ldots \ldots$. & $3.675-3.755$ & 4.06 & 13.03 \\
$1990 \ldots \ldots \ldots \ldots$. & $3.655-3.755$ & 4.08 & 13.05 \\
$1991 \ldots \ldots \ldots \ldots$. & $3.650-3.755$ & 4.08 & 13.10 \\
$1992.6 \ldots \ldots \ldots$. & $3.650-3.755$ & 4.08 & 13.11 \\
\hline & &
\end{tabular}

cate that $T_{\text {eff }} \sim 6500 \mathrm{~K}$ and $\log g \sim 1.5$, which corresponds to a F6-F7 supergiant.

We summarize the data for FG Sge in Table 5.

\subsubsection{V605 Aql}

Observations of V605 Aql, the central star of the planetary nebula Abell 58 (Ford 1971), are sparser than those of FG Sge and Sakurai's object. V605 Aql began brightening in 1917 September (Bidelman 1971) and reached a maximum photographic magnitude of $m_{\mathrm{pg}}=10.2$ in 1919 August (Wolf 1920). A secondary maximum occurred in 1921 (Lundmark 1921), and two more optical brightenings occurred in 1923 (Seitter 1987). Clayton \& de Marco (1997) have scanned a spectrum of V605 Aql obtained by Lundmark in 1921 during its secondary maximum. They describe it as being typical of a cool $\left(T_{\text {eff }} \sim 5000 \mathrm{~K}\right) \mathrm{R} \mathrm{CrB}$ star because of the presence of deep Swan bands of $C_{2}$ and violet bands of $\mathrm{CN}$, the characteristic absence of Balmer lines, the $G$ band of $\mathrm{CH}$, and ${ }^{12} \mathrm{C}^{13} \mathrm{C}$ bands. Hence V605 Aquilae shows evidence of severe hydrogen and ${ }^{13} \mathrm{C}$ deficiency. Assuming a distance of $3.5 \mathrm{kpc}$ and interstellar reddening of $A_{B}=2.3 \mathrm{mag}$, Clayton \& de Marco (1997) estimate that the object's luminosity in 1919 was $\sim 10^{4} L_{\odot}$. The photographic magnitude in 1917, $m_{\mathrm{pg}}=15$, corresponds to a blue luminosity of $\sim 50 L_{\odot}$. No temperature estimate has been published for V605 Aql for the year 1917. However, if we assume it was something comparable to that of $\mathrm{SO}$ $\left(\log T_{\text {eff }} \sim 4.0\right)$, then it can be deduced that the change in radius would be approximately from $\log R \sim 11.2$ to 12.97 .

The planetary nebula, Abell 58, around V605 Aql has a H-deficient central knot (Pottasch et al. 1986; Guerrero \& Manchado 1996). Spectra of the central knot clearly show
TABLE 6

Summary of V605 AQL Data From Clayton \& de Marco (1997) and Our Estimates

\begin{tabular}{cccc}
\hline \hline Date & $\begin{array}{c}\log T_{\text {eff }} \\
(\mathrm{K})\end{array}$ & $\log L / L_{\odot}$ & $\begin{array}{c}\log R \\
(\mathrm{~cm})\end{array}$ \\
\hline $1917 \ldots .$. & $\sim 4.0^{*}$ & $\sim 1.7^{*}$ & $11.2^{*}$ \\
$1919 \ldots .$. & $\sim 3.8$ & 4.0 & $13.0^{*}$ \\
$1992 \ldots .$. & $\sim 4.7$ & $\sim 4.0$ & $11.0^{*}$ \\
\hline
\end{tabular}

NoTE.-Our estimates are marked with an asterisk.

the presence of a broad C IV emission feature characteristic of a Wolf Rayet star. Clayton \& de Marco (1997) estimate that, if the $\mathrm{C}$ IV emission dominates the stellar spectrum, then V605 Aql currently has an effective temperature in excess of $50,000 \mathrm{~K}$.

Near infrared photometry of V605 Aql was obtained in 1992 by Harrison (1996). His analysis of this data and the IRAS fluxes indicates that V605 Aql is surrounded by a dusty shell that obscures a hot central source. The best fit to the optical-near-infrared flux distribution is a $50,000 \mathrm{~K}$ black body reddened by $A_{V}=9 \mathrm{mag}$. With this amount of extinction, V605 Aql may have a bolometric luminosity close to that of the maximum in 1919. If this is indeed the case, we would find for the present time $\log R \sim 11.0$. We summarize these results in Table 6 .

\subsection{General Comparisons to Model Parameters}

In Table 7 we present a comparison of observed parameters of the born-again objects with select models from our grid. Data listed for models are taken from the evolutionary stage in which is the very late helium flash is under way. The planetary nebula kinetic age for models is the time from photoionization of the planetary nebula to the beginning of the VLTP. This comparison shows that the kinetic age timescales, luminosity, and radii for our models are the right order.

\subsection{A Solution of the Timescale Problem}

With this theoretical picture of very late helium flash behavior (which one could argue is actually a very late helium and hydrogen flash) for low convective efficiency, $\beta \sim 10^{-4}$, we are poised to answer an important and previously troubling question: Why has Sakurai's object, and apparently V605 Aql, been observed to evolve to cool temperatures in about 4-6 yr (Clayton \& de Marco 1997; Asplund et al. 1997), while FG Sge has clearly been observed to be cooling for some $120 \mathrm{yr}$ (van Genderen \& Gautschy 1995)? In Figure 5 we compare how radii change as a function of time for Sakurai's object, FG Sge, V605 Aql, and an evolutionary model. This comparison shows clearly that a possible solution to the timescale problem is that Sakurai's object and FG Sge are at different stages of a common very late flash evolution. Though the observations for SO are sparse, the slope of the curve resembles closely the first and much faster (4.5-8.5 yr) approach to cooler temperatures (and larger radius). The slope, shape, and timescale for FG Sge unmistakably resembles the second, slower (200-550 yr) approach to cooler temperatures and giant radius. If this explanation of the difference in evolutionary timescales of the objects is correct, we can predict that SO will increase in temperature (along the top of the 
TABLE 7

COMPARISON WITH OBSERVATIONS

\begin{tabular}{|c|c|c|c|c|}
\hline $\begin{array}{c}\text { Model Metallicity } \\
\text { or Object }\end{array}$ & $\begin{array}{c}\text { H-R Diagram } \\
\text { Crossing Tmes } \\
\text { (yr) }\end{array}$ & $\begin{array}{c}\text { Kinetic Age of } \\
\text { Planetary Nebulae } \\
(\mathrm{yr})\end{array}$ & $\begin{array}{l}\text { Maxima in } \\
\text { Luminosity } \\
\left(10^{4} L_{\odot}\right)\end{array}$ & $\begin{array}{l}\text { Maxima in Radius } \\
\qquad\left(10^{12} \mathrm{~cm}\right)\end{array}$ \\
\hline$Z=0.001 \ldots \ldots \ldots \ldots \ldots$ & $4.0,9.7,240$ & 13,000 & $1.27,1.27$ & $5.39,0.57$ \\
\hline$Z=0.004 \ldots \ldots \ldots \ldots \ldots$ & $4.7,12,340$ & 29,000 & $1.51,1.07$ & $7.85,6.09$ \\
\hline$Z=0.010 \ldots \ldots \ldots \ldots \ldots$ & $6.0,21,470$ & 9,000 & $1.40,0.88$ & $8.66,6.96$ \\
\hline$Z=0.020 \ldots \ldots \ldots \ldots \ldots$ & $7.5,26,480$ & 16,000 & $1.32,0.75$ & $9.13,5.88$ \\
\hline Sakurai's object...... & $\sim 6.0$ & $3400-5600^{a}$ & $1.0^{\mathrm{b}}$ & $6.3^{\mathrm{b}}$ \\
\hline V605 Aql.................. & $\sim 2.0^{\mathrm{c}}$ & $1300-3500^{\mathrm{c}}$ & $\sim 1.0^{\mathrm{c}}$ & $9.1^{\mathrm{c}}$ \\
\hline FG Sge................... & $\sim 120.0$ & $6000-10,000^{\mathrm{d}}$ & $1.20^{\mathrm{e}}$ & $12.6^{\mathrm{e}}$ \\
\hline
\end{tabular}

a From Kimeswenger \& Kerber 1998.

${ }^{\mathrm{b}}$ From Duerbeck et al. 1997.

c From Clayton \& de Marco 1997.

d From Blöcker \& Schönberner 1997.

e From van Genderen \& Gautschy 1995.

loop in the H-R diagram) in a slightly slower timescale (20$50 \mathrm{yr}$ ) than it decreased. Following this increase it would again brighten and cool gradually (200-500 yr), in the same fashion as has been observed for FG Sge. It is expected that it will spend approximately $50-100 \mathrm{yr}$ on the warmer side of the very late giant branch before making this final approach to the AGB. Though the data for V605 Aql are incomplete, they adds important corroborative evidence. We can say with confidence that it underwent a flash in 1917, that it cooled and grew to giant size on roughly the same timescale as SO, and recent observations indicate that the central star is roughly the same luminosity and has an effective temperature $T_{\text {eff }}>50,000 \mathrm{~K}$ (Clayton \& de Marco 1997). Now, noting that its minimum temperature had been reached 82 yr ago, V605 Aql provides a single and perfect example of a link between SO and FG Sge. Specifically, our models evolve from cooler to warmer temperatures at roughly constant luminosity (dropping about $0.5 \mathrm{mag}$ ) and shrink to

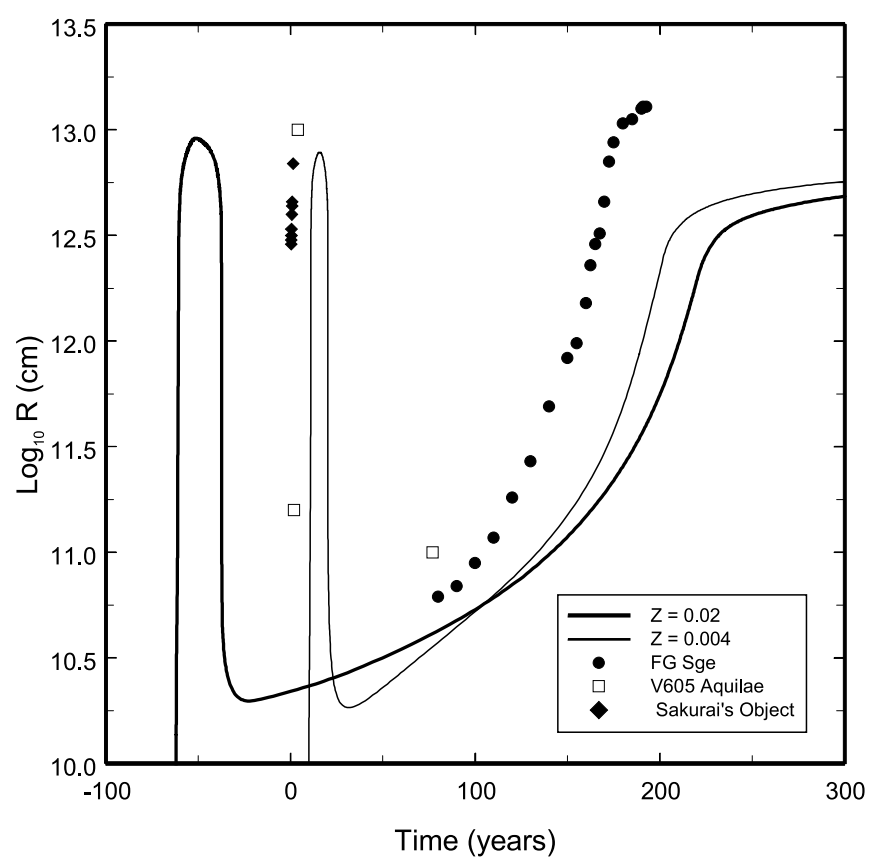

FIG. 5.-Comparison of radius estimates from the observations of FG Sge, V605 Aql, and Sakurai's object with model radii as a function of time. approximately solar size with a timescale between 20 and 50 yr (depending on metallicity), as is the case for V605 Aql. The sparse data for V605 Aql is also shown in Figure 5. Remarkably, each of the three observed objects represents one of the three crossings of the H-R diagram: from hot to cool (SO), from cool to hot (V605 Aql), and finally back to a cool giant (FG Sge).

\subsection{Chemical Abundances}

The most complete analysis of the abundances of a bornagain star is that of Asplund et al. (1999), who derived abundances for Sakurai's object from spectra obtained in 1996. They find that the abundances of many elements, including $\mathrm{He}, \mathrm{C}, \mathrm{N}$, and O remained roughly constant from 1996 April to October, but $\mathrm{H}$ decreased in abundance, and many $s$ process elements increase in abundance over this period. In Table 8 we compare, in mass fractions, the surface abundance composition for SO in 1996 April and October with those of our very late flash models at point $\mathrm{E}$ in the H-R diagram. From the abundances of $\mathrm{Fe}$ and $\mathrm{Ni}$, we can infer that metallicity of the progenitor star was closer to $0.001-0.004$ than to $0.01-0.02$. There are significant differences between the model surface hydrogen abundances and those observed. Also the observed Ne abundance is larger than that of the models. However, there is reasonably good agreement with the $\mathrm{CNO}$ abundances. In particular the $\mathrm{C} / \mathrm{O}$ ratios of the models are well within the error bars of the observed ratios. This is in contrast to Iben \& MacDonald (1995), who found $X_{\mathrm{C}} \sim 0.15, X_{\mathrm{N}} \sim 0.05$, and $X_{\mathrm{O}} \sim 0.01$ at a similar stage of evolution. By comparing reaction rates at the relevant temperatures and densities, we find that our triple-alpha rate is, in general, lower than that used by Iben \& MacDonald (1995), but our ${ }^{12} \mathrm{C}(\alpha, \gamma){ }^{16} \mathrm{O}$ rate is higher by about $50 \%$. This suggests that the difference in oxygen abundance is due to differences in reaction rates. Better agreement with the surface $\mathrm{H}$ abundance is obtained with higher mixing efficiency but then the $\mathrm{CNO}$ abundances are too high.

\section{CONCLUSIONS AND DISCUSSION}

In this paper we present the results of a number of calculations of the evolution of $1 M_{\odot}$ stars with emphasis on models relevant to the investigation of the born-again stars. We find that the light curves of Sakurai's object, V605 Aql, 
TABLE 8

Comparison of Model Surface Abundances (in Mass Fractions) with Those of SO

\begin{tabular}{|c|c|c|c|c|c|c|c|}
\hline Abundance & $\mathrm{H}$ & $\mathrm{He}$ & $\mathrm{C}$ & $\mathrm{N}$ & $\mathrm{O}$ & Total Other & $\mathrm{C} / \mathrm{O}$ \\
\hline$Z=0.001 \ldots$ & 0.680 & 0.304 & 0.0093 & 0.0020 & 0.0030 & 0.0012 & 3.1 \\
\hline$Z=0.004 \ldots \ldots \ldots \ldots \ldots \ldots \ldots$ & 0.478 & 0.453 & 0.045 & 0.0054 & 0.013 & 0.0047 & 3.5 \\
\hline$Z=0.010 \ldots \ldots \ldots \ldots \ldots \ldots$ & 0.389 & 0.472 & 0.094 & 0.0043 & 0.031 & 0.0090 & 3.0 \\
\hline$Z=0.020, \beta=10^{-4} \ldots \ldots$. & 0.355 & 0.475 & 0.116 & 0.0064 & 0.033 & 0.014 & 3.5 \\
\hline$Z=0.020, \beta=10^{-2} \ldots \ldots$. & 0.041 & 0.544 & 0.280 & 0.031 & 0.075 & 0.029 & 3.7 \\
\hline SO in 1996 Apr............. & 0.0086 & 0.863 & 0.052 & 0.012 & 0.020 & 0.043 & 2.6 \\
\hline SO in 1996 Oct .............. & 0.00084 & 0.840 & 0.063 & 0.0093 & 0.034 & 0.053 & 1.9 \\
\hline
\end{tabular}

and FG Sge can all be explained in a single model in which the convective mixing efficiency is substantially reduced below that obtained from standard mixing-length theory. In this case, the born-again stars follow a double-loop path in the H-R diagram in which they first evolve quickly to the AGB, return to the blue, and then evolve more slowly back to the AGB for a second time before finally returning to the white dwarf cooling track. The observed timescale differences result from Sakurai's object having evolved to the AGB for the first time, whereas FG Sge was observed during its second return to the AGB.

The "double-loop" evolution is due to the initiation of shell hydrogen burning when protons are ingested by the outward growing convection zone above the helium shell source. Energy produced by hydrogen burning is responsible for the first expansion to the AGB. The star contracts when hydrogen burning is quenched and then reexpands as a result of the rekindling of the helium shell source. A critical aspect of the double-loop evolution is the requirement that the convective mixing efficiency be much lower than predicted by standard mixing-length theory. Too efficient mixing leads to a completely homogenized envelope with a very low hydrogen abundance. A giant configuration is then not possible for our stars with low core masses (Trimble \& Paczyński 1973; Weiss 1987) and the second expansion to red giant dimensions does not occur. This is the probable reason that the calculations of Herwig et al. (1999) and Herwig (2001) with a higher mixing efficiency produce a single loop in the H-R diagram rather than the double loop that we find for lower mixing efficiency.

Our low convective mixing efficiency model allows us to make some testable predictions for the future evolution each of the three objects. Clayton \& de Marco (1997) have suggested that V605 Aql may be a glimpse into the future of SO. Our calculations supports that prediction and also indicate that FG Sge is showing us the future of both SO and V605 Aql. Specifically, we expect SO to move to warmer temperatures in the next 20-50 yr and that it then will resemble V605 Aql's present state (and this should be soon observable), and we expect V605 Aql to cool back toward the AGB but not noticeably for as much as $50-70 \mathrm{yr}$, at which time it will evolve in the same way as has been observed for FG Sge for the last 120 yr. Finally, FG Sge will show signs of increasing effective temperature by about $1500-2000 \mathrm{~K}$ in as soon as $10-20 \mathrm{yr}$, depending on the metallicity of its progenitor star.

When a born-again star leaves the AGB for the last time, it will first become a H-deficient central star of a planetary nebula and then evolve through the region of the H-R diagram populated by the PG 1159 stars (Iben et al. 1983; Werner 2001). In Table 9 we compare the model surface abundances (in mass fractions) at the time of maximum effective temperature before the white dwarf cooling sequence, for $\beta=10^{-4}$, with those of the H-deficient CSPN of spectral type [WCE] (Koesterke \& Hamann 1997) and [WCL] (Leuenhagen \& Hamann 1998) and the PG 1159 stars (Dreizler \& Heber 1998). In general, the model He and $\mathrm{N}$ abundances are larger, and the $\mathrm{C}$ and $\mathrm{O}$ abundances smaller, than the average observed abundances. We surmise that either the progenitors of the PG 1159 stars and the Hdeficient CSPN have a higher convective mixing efficiency than the progenitors of the born-again stars or we have underestimated the mass-loss rate for the CSPN phases of evolution. The first case will lead to a greater dredge-up of $\mathrm{C}$ and $\mathrm{O}$, and the burning of more $\mathrm{H}$ and $\mathrm{N}$, whereas the second case can lead to same effect by stripping off the $\mathrm{H}$ and $\mathrm{N}$ rich surface layers. We note however that the model $\mathrm{C} / \mathrm{O}$ ratios are consistent with the observations, without the need to include convective overshoot as had been suggested by Herwig et al. (1999). A clear indication of whether FG Sge and Sakurai's object will evolve to become H-deficient CSPN and then PG 1159 stars would be the detection of $s$ process elements in the photospheres of these objects. Until then, the specific question of whether FG Sge and Sakurai's object will evolve through these stages remains open.

Our model for the evolution of the born-again stars depends crucially on the convective mixing efficiency being many orders of magnitude below that of standard mixinglength theory. Possible mechanisms for a reduction in convective efficiency are the effects of molecular weight gradients (Ledoux 1947; Kato 1966) and magnetic fields (Gough \& Tayler 1966; Moss 1968; Mullan \& MacDonald 2001). A stabilizing molecular weight gradient is predicted to be present in the envelope of an AGB star as a result of hydrogen burning. Magnetic fields in AGB stars have been proposed as a means of shaping planetary nebulae

TABLE 9

Abundance COMParison With the (WC) AND PG 1159 Stars

\begin{tabular}{|c|c|c|c|c|c|}
\hline Abundance & $\mathrm{H}$ & $\mathrm{He}$ & $\mathrm{C}$ & $\mathrm{N}$ & $\mathrm{O}$ \\
\hline$Z=0.004 \ldots \ldots \ldots \ldots \ldots \ldots \ldots$ & 0.014 & 0.68 & 0.18 & 0.045 & 0.058 \\
\hline$Z=0.01$ & 0.039 & 0.59 & 0.26 & 0.018 & 0.083 \\
\hline$Z=0.02 \ldots \ldots \ldots \ldots \ldots \ldots$ & 0.065 & 0.57 & 0.26 & 0.011 & 0.067 \\
\hline$(\mathrm{WCL})^{\mathrm{a}} .$. & 0.036 & 0.42 & 0.50 & & 0.062 \\
\hline 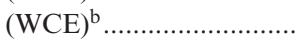 & & 0.56 & 0.30 & 0.005 & 0.14 \\
\hline PG 1159 nonpulsators ${ }^{\mathrm{c}} \ldots$ & & 0.65 & 0.28 & 0.0 & 0.073 \\
\hline PG 1159 pulsators ${ }^{c}$.......... & & 0.40 & 0.43 & 0.014 & 0.16 \\
\hline
\end{tabular}

a From Leuenhagen \& Hamann 1998.

b From Koesterke \& Hamann 1997.

c From Dreizler \& Heber 1998. 
(Chevalier \& Luo 1994; Garcia-Segura \& Lopez 2000), and their presence has been inferred for U Her from the polarization of the $\mathrm{OH}$ maser emission (Palen \& Fix 2000). Any reduction in convective mixing efficiency by molecular weight gradients or magnetic fields is expected to be accompanied by a reduction in convective energy flux. Further work is necessary to determine how this will influence the evolution of the models.

A relevant question is whether a significantly reduced convective mixing efficiency could be present on other parts of the H-R diagram. The effects of a reduction in convective mixing efficiency might be expected to show up at evolutionary phases where convective dredge-up occurs. However a study of red giant branch stars shows that there is no significant difference in the evolution of their photospheric properties, including predicted surface abundance changes, between calculations with $\beta=1$ and $\beta=10^{-4}$. Hence, a relatively low convective mixing efficiency could be quite common. We stress here that we are differentiating between convective mixing eficiency and the effects of processes such as convective overshoot and convective penetration, which appear necessary to explain the abundance patterns seen in RGB stars.

We thank Dermott Mullan for interesting and useful discussions.
Abbott, D. 1982, ApJ, 259, 282

Alexander, D. R., \& Ferguson, J. W. 1994, ApJ, 437, 879

Arkhipova, V. P. 1994, Pis'ma Astron. Zh., 20, 919

Arnett, D. 1996, Supernovae and Nucleosynthesis (Princeton: Princeton Univ. Press)

Asplund, M. 1998, A\&A, 330, 641

Asplund, M., Gustafsson, B., Lambert, D. L., \& Rao, N. K. 1997, A\&A, $321, \mathrm{~L} 17$

Asplund, M., Gustafsson, B., Rao, N. K., \& Lambert, D. L. 1998, A\&A, 332,651

Asplund, M., Lambert, D. L., Kipper, T., Pollacco, D., \& Shetrone, M. D. 1999, A\&A, 343, 507

Beaudet, G., Petrosian, V., \& Salpeter, E. E. 1967, ApJ, 150, 979

Bidelman, W. P. 1971, ApJ, 165, L7

Blöcker, T. 2001, Ap\&SS, 275, 1

Blöcker, T., \& Schönberner, D. 1997, A\&A, 324, 991

Bond, H. E., Meakes, M. G., Liebert, J. W., \& Renzini, A. 1993, in

IAU Symp. 155, Planetary Nebulae, ed. R. Weinberger \& A. Acker (Dordrecht: Kluwer), 499

Boublik, T. 1970, J. Chem. Phys., 53, 471

Bragaglia, A., Renzini, A., \& Bergeron, P. 1995, ApJ, 443, 735

Caughlan, G. R., \& Fowler, W. A. 1988, At. Data Nucl. Data Tables, 40, 283

Chevalier, R. A., \& Luo, D. 1994, ApJ, 421, 225

Clayton, G. C., \& de Marco, O. 1997, AJ, 114, 2679

Dreizler, S., \& Heber, U. 1998, A\&A, 334, 618

Dreizler, S., \& Werner, K. 1996, in ASP Conf. Ser. 96, Hydrogen Deficient

Stars, ed. C. S. Jeffery \& U. Heber (San Francisco: ASP), 281

Duerbeck, H., Benetti, S., Gautschy, A., van Genderen, A. M., Kemper, C., Liller, W., \& Thomas, T. 1997, AJ, 114, 1657

Duerbeck, H., Hazen, M. L., Misch, A. A., \& Seitter, W. C. 2002, Ap\&SS, 279,183

Eggleton, P. P. 1971, MNRAS, 151, 351 1972, MNRAS, 156, 361

Eggleton, P. P., Faulkner, J., \& Flannery, B. P. 1973, A\&A, 23, 325

Fontaine, G., Graboske, H. C., Jr., \& van Horn, H. M. 1977, ApJS, 35, 293

Ford, H. C. 1971, ApJ, 170, 547

Fowler, W. A., Caughlan, G. R., \& Zimmerman, B. A. 1975, ARA\&A, 13, 69

Fujimoto, M. Y. 1977, PASJ, 29, 331

García-Berro, E., \& Iben, I. 1994, ApJ, 434, 306

Garcia-Segura, G., \& Lopez, J.A. 2000, ApJ, 544, 336

Gonzalez, G., Lambert, D. L., Wallerstein, G., Rao, N. K., Smith, V. V., \& McCarthy, J. K. 1998, ApJS, 114, 133

Gough, D. O., \& Tayler, R. J. 1966, MNRAS, 133, 85

Guerrero, M. A., \& Manchado, A. 1996, ApJ, 472, 711

Haft, M., Raffelt, G., \& Weiss, A. 1994, ApJ, 425, 222

Harris, M. J., Fowler, W. A., Caughlan, G. R., \& Zimmerman, B. A. 1983, ARA\&A, 21, 165

Harrison, T. E. 1996, PASP, 108, 1112

Herbig, G. H., \& Boyarchuk, A. A. 1968, ApJ, 153, 397

Herwig, F. 2001, ApJ, 554, L71

Herwig, F., Blöcker, T., Langer, N., \& Driebe, T. 1999, A\&A, 349, L5

Hollowell, D., Iben, I., \& Fujimoto, M. Y. 1990, ApJ, 351, 245

Iben, I. 1976, ApJ, 208, 165

1982, ApJ, 260, 82

1984, ApJ, 277, 333

Iben, I., Fujimoto, M. Y., \& MacDonald, J. 1992, ApJ, 388, 521

Iben, I., Kaler, J. B., Truran, J. W., \& Renzini, A. 1983, ApJ, 264, 605

Iben, I., \& Livio, M. 1993, ApJ, 406, L15

Iben, I., \& MacDonald, J. 1995, in White Dwarfs, Proc. 9th European

Workshop on White Dwarfs, ed. D. Koester \& K. Werner (Lecture

Notes in Physics, Vol. 443; Berlin: Springer), 48

Iglesias, C. A., \& Rogers, F. J. 1996, ApJ, 464, 943
Itoh, N., Totsuji, H., Ichimaru, S., \& Dewitt, H. E. 1979, ApJ, 234, 1079

Jimenez, R., Jorgensen, U. G., Thejll, P., \& MacDonald, J. 1995, MNRAS, 275,1245

Jurcsik, J. 1993, Acta Astron., 43, 353

Kato, S. 1966, PASJ, 18, 374

Kerber, F., Koppen, J., Roth, M., \& Trager, S. C. 1999, A\&A, 344, L79

Kimeswenger, S., \& Kerber, F. 1998, A\&A, 330, L41

Kipper, T. 1996, in ASP Conf. Ser. 96, Hydrogen-deficient Stars, ed. C. S. Jeffery \& U. Heber (San Francisco: ASP), 329

Kipper, T., \& Klochkova, G. 1997, A\&A, 324, L65

Koesterke, L., \& Hamann, W.-R. 1997, A\&A, 320, 91

Kudritzki, R. P., \& Reimers, D. 1978, A\&A, 70, 227

Kwok, S. 1987, in Late Stages of Stellar Evolution, ed. S. Kwok \& S. R. Pottasch (Dordrecht: Kluwer), 321

Langer, G. E., Kraft, R. P., \& Anderson, K. S. 1974, ApJ, 189, 509

Lebowitz, J. E., Helfand, E., \& Praestgaard, J. 1965, J. Comput. Phys., 43, 774

Ledoux, P. 1947, ApJ, 105, 305

Leuenhagen, U., \& Hamann, W.-R. 1998, A\&A, 330, 265

Lundmark, K. 1921, PASP, 33, 314

Mansoori, G. A., Carnahan, N. F., Starling, K. F., \& Leland, T. W. 1971, J. Comput. Phys., 54, 1523

Mihalas, D. 1970, Stellar Atmospheres (San Francisco: Freeman)

Montesinos, B., Cassatella, A., Gonzalez-Riestra, R., Fernandez-Castro, T., Eiroa, C., \& Jimenez-Fuensalida, J. 1990, ApJ, 363, 245

Moss, D. L. 1968, MNRAS, 141, 165

Mullan, D. J. \& MacDonald, J. 2001, ApJ, 559, 353

Nakano, S., Benetti, S., \& Duerbeck, H. W. 1996, IAU Circ. 6322

Napiwotzki, R. 1999, Ä\&A, 350, 101

Nomoto, K. Thielemann, F.-K, \& Miyaji, S. 1985, A\&A, 149, 239

Palen, S., \& Fix, J. D. 2000, ApJ, 531, 391

Pottasch, S. R., Mampaso, A., Manchado, A., \& Menzies, J. 1986, in IAU Colloq. 87, Hydrogen-deficient Stars and Related Objects, ed. K Hunger, D. Schönberger, \& N. Kameswara Rao (Dordrecht: Reidel), 359

Ramadurai, S. 1976, MNRAS, 176, 9

Reimers, D. 1975, Mem. Soc. R. Sci. Liège, 8, 369

Ritossa, C., García-Berro, E., \& Iben, I. 1999, ApJ, 515, 381

Salpeter, E. E., \& van Horn, H. M. 1969, ApJ, 155, 183

Schönberner, D. 1979, A\&A, 79, 108

Schwarzschild, M., \& Härm, R. 1965, ApJ, 142, 855

Seitter, W. C. 1987, Messenger, 50, 14

Shetrone, M. D., \& Keane, M. 1997, in The Third Conference on Faint Blue Stars, ed. A. G. D. Philip, J. Liebert, R. Saffer, \& D. S. Hayes (Schenectady: Davis), 505

Smolinski, J., Climenhaga, J. L., \& Kipper, T. 1976, PASP, 88, 67

Stringfellow, G. S., DeWitt, H. E., \& Slattery, W. L. 1990, Phys. Rev. A, 41,1105

Trimble, V., \& Paczyński, B. 1973, A\&A, 22, 9

van Genderen, A. M. \& Gautschy, A. 1995, A\&A, 294, 453

Vennes, S. 1999, ApJ, 525, 995

Weigert, A. 1967, in Colloquium on Late-Type Stars, ed. M. Hack (Trieste: Osserv. Astron.), 388

Weiss, A. 1987, A\&A 185, 165

Wentzel, W., \& Fürtig, W. 1967, Sterne, 43, 19

Werner, K. 2001, Ap\&SS, 275, 27

Werner, K., Dreizler, S., Heber, U., \& Rauch, T. 1996, in ASP Conf. Ser. 96, Hydrogen-deficient Stars, ed. C. S. Jeffery \& U. Heber (San Francisco: ASP), 267

Winkler, K.-H. A., Norman, M. L., \& Newman, M. J. 1984, J. Phys. D, 12, 408

Wolf, M. 1920, AN, 211, 119

Wood, P. R., \& Cahn, J. H. 1977, ApJ, 211, 499

Woodward, C. E., Lawrence, G. F., Gehrz, R. D., Jones, T. J., Kobulnicky, H. A., Cole, J., Hodge, T., \& Thronson, H. A. 1993, ApJ, 408, L37 